\title{
Behavioural aspects of reproduction in Chiroptera
}

\author{
J. W. Bradbury \\ Department of Biology, University of California, San Diego, La Jolla, California 92093, U.S.A.
}

The preceding papers in this Symposium have emphasized the diversity of chiropteran reproductive physiology. In many cases, these differences between species can be correlated with either differences in habitat and climate or differences in phylogenetic affinities. I should like to complete this portrait by focusing on reproductive differences within habitats and within species. While one tends to ignore variation at these levels when looking for broad patterns, it clearly exists and is potentially useful in elucidating the evolution of the general trends. To illustrate this point, I shall present several examples in which the broader habitat-specific or species-specific patterns are 'fine-tuned' at the individual or local level. This fine-tuning appears to be generated by interactions between the physiological potentials of each form, access to crucial resources, and current status in the ambient social milieu. My examples all involve timing, either with respect to age or to season, with which reproductive effort is undertaken. They include (a) a suite of related species sharing a common habitat and climate but showing diversity in reproductive timing, (b) intersexual differences in timing within a single species, and (c) intrasexual differences in timing. I shall use examples with which I am most familiar, but I suspect that these are representative of Chiroptera as a whole.

\section{Within-habitat diversity in timing}

As an example of within-habitat diversity, I shall summarize the reproductive patterns of four species of emballonurid bats which we have studied in western Costa Rica (Bradbury \& Vehrencamp, 1976, 1977b). These bats share a common roosting habitat and may even share a common roosting tree. They all experience a common climate of temperature and rainfall with a pronounced dry season from January to early May and a rainy season from late May through December. The bats are all aerial insectivores and weigh less than $10 \mathrm{~g}$.

The smallest species, Rhynchonycteris naso, roosts in colonies of 5 to 50 bats on the exposed boles of trees. In western Costa Rica, its parturition is loosely seasonal: most females in most colonies produce a single young in the late dry season (April-May), and a few females in each colony produce a second offspring in the late rainy season (October-November). However, there is marked asynchrony both between and within colonies, and lactating females can usually be found throughout the rainy season. Youngsters disperse to other colonies at 2-4 months of age and there is no sexual bias in dispersal rates or distances.

Bats of the species Saccopteryx leptura roost in similar sites but occur in colonies of only 19 animals. Parturition is much more seasonal, with a highly synchronized birth peak at the onset of the rains (early May). A few females produce a second young in late rainy season as well (November). Dispersal of the young may be delayed for up to 1 year in this species. Retention of young is not sex dependent.

Balantiopteryx plicata is a highly social emballonurid which favours well-lit caves, cliff faces, and boulder fields as diurnal roosts. In western Costa Rica, all females breed once only each year, and this parturition is highly synchronous (June). Parturition rates are high, with only $3 \%$ of the females examined failing to have young. Dispersal occurs at 2-3 months of age and there appear to be no sex biases in dispersal. 
Bats of the largest species, Saccopteryx bilineata, are found within hollow trees or between the buttresses of large trees. Colony size ranges from 2 to 50 bats per site. A single synchronous birth peak occurs each year at the onset of the rainy season (May). While nearly all females examined in March and April are found to be pregnant, an average $17 \%$ of these fail to produce a suckling youngster. Whether these fetuses are aborted or resorbed is still unknown. Dispersal occurs at 3 months of age and is highly sex biased: while nearly all juvenile females move to other colonies, an average $76 \%$ of male offspring remain near to or within the parental colony (Tannenbaum, 1975).

In spite of roosting in a common habitat, these four species exhibit considerable diversity in the timing and execution of reproductive activities. In several species, e.g. $R$. naso and $S$. leptura, roosts are so similar that differential roost dispersion or differences in microclimate during the day cannot explain divergences in reproductive behaviour. We have therefore examined nocturnal behaviour, in particular foraging, to try to explain the differences between the species. On the basis of following marked animals at night and monitoring insect food abundances, it is clear that the four species show marked divergences in their foraging behaviour: $R$. naso feeds exclusively over rivers, $S$. leptura is restricted to riparian forests, $B$. plicata favours open fields and deciduous forests, and $S$. bilineata moves between all of these habitats successively as insects peak in abundance.

Given the classical wisdom that animals often synchronize reproduction with food peaks, we surmised that the differences between these four species could be explained by seasonalities in the food in each habitat. In Text-fig. 1, we summarize our estimates of insect biomasses (dry weight) available to each species throughout the year and the observed timing of reproduction. Where several habitats are used, values are normalized for relative areas of each habitat and for bat body weights (see Bradbury \& Vehrencamp, 1977b).

The issue which immediately arises on viewing Text-fig. 1 is which phase of reproduction is to be synchronized with a food peak? Since gestation is 5 months long in these bats, lactation takes from 2-4 months, and dispersal can take up to 1 year, it is impossible to synchronize all phases of reproduction with a food maximum. When gestation occurs during a food peak, the stress on the female parent is minimized, although increased stress on the young during lactation and dispersal may be the consequence. Similarly, if lactation and/or dispersal occur during maximum food levels, the female parent suffers stress by having low food abundances during gestation. We have called the former situation a "parent-biased" timing and the latter a "progeny-biased" timing.

In these four species, there appears to be a gradient ranging from extremely parent-biased timing in $R$. naso, through an intermediate condition in $S$. leptura, to the strongly progeny-biased timings of $B$. plicata and $S$. bilineata. Specifically, Text-fig. 1 indicates that $R$. naso females encounter a food peak during the one period of the year that any synchrony in gestation occurs. The subsequent lactation and dispersal periods coincide with low food levels. Dispersal costs are significant because no young are retained within parental colonies. In $S$. leptura, the first and major parturition period occurs with average food levels during gestation, a peak during lactation, and low levels during what would be dispersal in the other 3 species. Unlike the other species, young do not disperse at 2-4 months of age, but remain with their parents for up to 1 year. Since all colony members share a common feeding territory, retention of young is costly to the parents in terms of insect food which could be used to produce additional young. Whether this is the reason why so few females produce a second youngster in November is still unknown. While insect abundances are not shown in Text-fig. 1 for $B$. plicata, the access to food of this species is similar to that shown for $S$. bilineata (Lopez-Forment, 1976). Both species experience food shortages during gestation but peak food levels during lactation and dispersal. The cost to the female of such timing is partly ameliorated in $S$. bilineata through what appears to be facultative abortion or resorption. This option does not seem to be available to $B$. plicata females. 

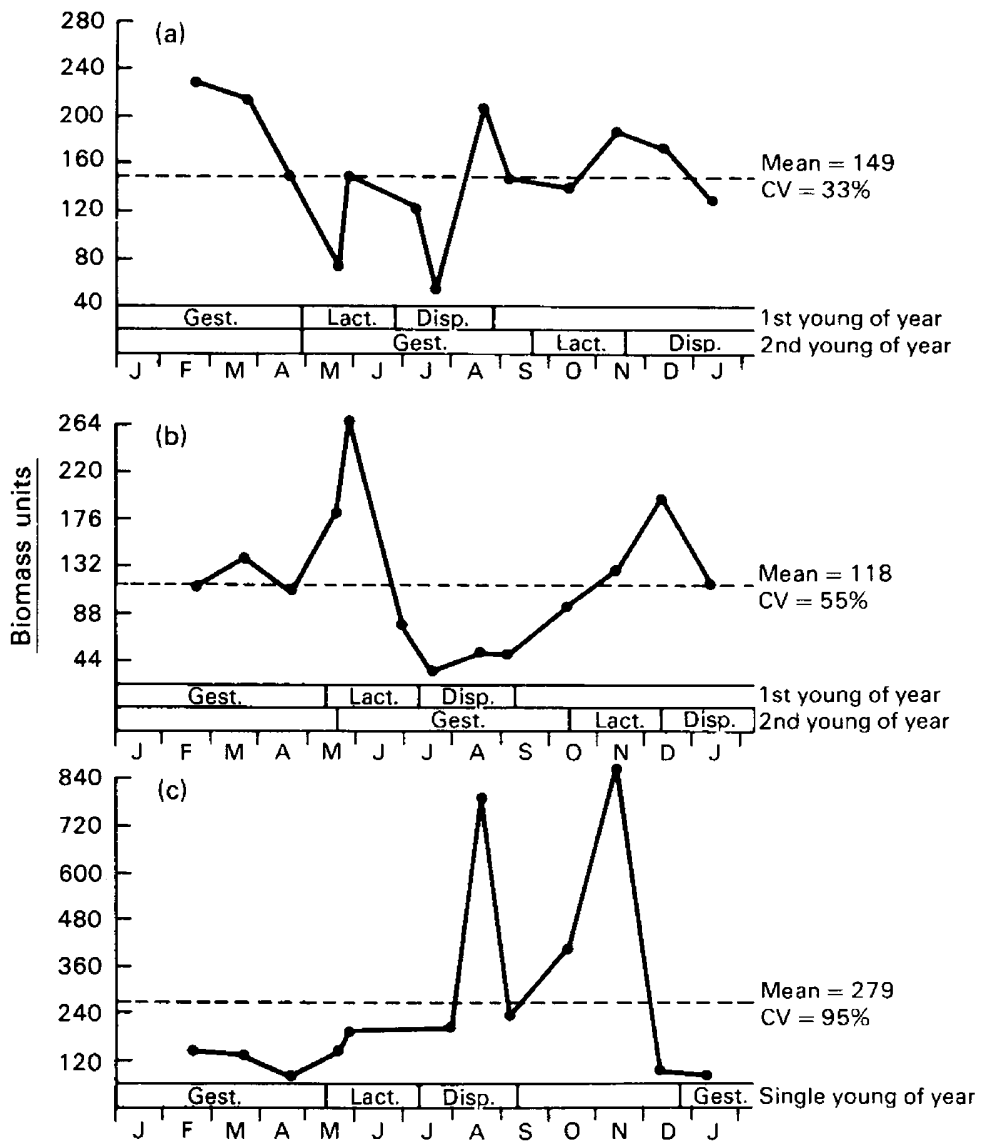

Text-fig. 1. Seasonal variation in food available to three emballonurid species (a, Rhynchonycteris naso; b, Saccopteryx leptura; c, S. bilineata) in relation to observed cycles. Vertical axis is estimated prey abundance in biomass units. The latter were computed from insect abundances in each habitat, allometric relationships of insect length and insect biomass, type of habitat utilized by each bat species in each season, and relative areas of each habitat type. CV, coefficient of variation; Gest. = gestation; Lact. = lactation; Disp. = dispersal of young. For further details, see Bradbury \& Vehrencamp (1977b).

The above ranking of parental risks is matched well by our estimates of (1) the magnitude of seasonal variations in food abundance for each species and (2) our estimates of annual adult mortality. As seen in Text-fig. 1, seasonal variations in food abundance range from $33 \%$ for $R$. naso to $95 \%$ for $S$. bilineata (as measured by monthly coefficients of variation). Values for $B$. plicata are as high or higher than those for $S$. bilineata (Lopez-Forment, 1976). Since we believe access to food to be a major determinant of adult survival (see Bradbury \& Vehrencamp, 1976), these variations in food levels ought to be reflected in adult survival rates. This appears to be the case. Our estimates of annual adult survival for female $R$. naso are $80 \%$ or higher, for $S$. bilineata an average $78 \%$, and for $B$. plicata $54 \%$. Estimates for $S$. leptura females appear to be better than $80 \%$, but our samples are small.

The parallel rankings of these species according to increasing progeny-bias in reproductive timing, adult female mortality rates, and seasonal variability in food supplies suggest the following interpretations. While all four species share similar roosts and climate, they exhibit marked divergence in their partitioning of available foraging sites. Inherent differences in the 
stability of food levels for each site generate differences in adult survival rates. These in turn dictate whether reproductive timing should be parent- or progeny-biased, since an adult with little chance of survival should put all effort into any given reproduction whereas an adult with a long remaining life-span should take few risks for any given offspring (Goodman, 1974; Schaffer, 1974).

There are two general points to be drawn from this example of within-habitat reproductive diversity. The first is that exposure to a common climatic cycle and use of a common roosting habitat are not sufficient conditions for reproductive convergence in bats. As long as the considered species can partition the critical resource sites, differences in the ways in which the same cycle will be perceived will arise and lead to different demographic strategies and timings. The second point is that ecological differences, e.g. in foraging, between related species in the same habitat may be sufficient to override any tendencies for reproductive convergence via phylogenetic affinity. In fact, it may be the case that related and sympatric species are the ones most likely to show local partitioning and hence diversity in reproductive timing, while unrelated species which can coexist in the same specific sites show convergence.

\section{Intersexual diversity in timing}

The most obvious differences in reproductive timing within species are to be found between the two sexes. While males and females must be seasonally synchronized for mating, this still leaves great latitude for differences between the sexes in (a) the age at first reproduction, and (b) the fraction of males or females within a given age class which undertake reproduction in a particular season. Sexual differences in age at maturity are known for a number of bats. Males appear to reach sexual maturity earlier than females in Megaderma lyra (Ramaswamy, 1961) and Plecotus auritus (Stebbings, 1966). The reverse is true in Macrotus waterhousii (Bradshaw, 1962), Tadarida brasiliensis (Short, 1961; Constantine, 1967) and Hypsignathus monstrosus (Bradbury, 1977a) among others. Differences in the fraction of adults of either sex mating each season have been noted rarely in the literature, but this probably reflects a lack of study rather than the absence of such differences. In this section, I shall present some field data on the large Hypsignathus monstrosus, showing that both kinds of inter-sexual difference exist, and that these appear to arise, as with emballonurids, as a function of sexual differences in demographic strategies, current social status, and access to crucial food supplies.

Hypsignathus monstrosus is a megachiropteran of the African lowland rain forests. Mating occurs twice annually during the two dry seasons typical of equatorial Africa. Where we have studied this species in Gabon (see Bradbury, 1977a), all mating occurs at traditional "lek" sites during the first half of the night. The leks consist of 20 to 135 males each and are spaced about $14 \mathrm{~km}$ apart. They typically are situated along the borders of rivers where each displaying male defends its own $10 \mathrm{~m}$ diameter mating territory. Males display from dusk to midnight and again before dawn by flapping their wings and emitting a loud monotonous honking. Females visit these male assemblies, examine a succession of males, and eventually select one for mating. As with most other avian and mammalian lek systems, a few males at each site do most of the mating.

Sexual differences in the undertaking of reproductive effort are pronounced in this species. With regard to age at maturity, we have found that both captive and wild females can become pregnant at 6 months of age and bear their first offspring as yearlings. Males mature much more slowly and until 12 months of age are distinguished from adult females only with difficulty. Between 12 and 18 months of age, males change rapidly from a female-like shape to the adult male form with its enlarged muzzle, hypertrophied larynx and $60 \%$ greater body weight. Active spermatozoa can only be found in the testes of males 18 months or older. Males therefore take three times longer to become sexually mature than do females. 
Participation rates in a given reproductive season also vary between the two sexes. An average of $78 \%$ of all yearling females and $95 \%$ of all older females are pregnant or lactating in any given breeding season. Many of the yearlings which are not pregnant or lactating show enlarged teats indicating that a pregnancy was initiated but terminated early for some reason. In contrast, many of the adult males which we have marked or radio-tracked do not take up display stations at all in a given season, or if they do, display for only a short period. Even those males which display regularly and achieve copulations will periodically abandon their perches and spend the night foraging or roosting alone. There is considerable synchrony both within and between leks in male absentee behaviour, and this appears to correlate with periods of low ambient temperatures. During one study period in 1974 , minimum temperatures of $11-15^{\circ} \mathrm{C}$ were coincident with a complete abandonment of the lek by displaying males. This occurred even though copulations had begun a week earlier and females were observed to visit the site. When temperatures returned to the more typical values of $17^{\circ} \mathrm{C}$ or more, male display resumed where it had broken off.

It appears that in Hypsignathus the undertaking of reproduction occurs early and regularly in females, but is delayed and facultative in males. Some of these differences may arise from the pronounced sexual dimorphism of the species. At least in captivity, males and females grow in weight at similar rates, and the larger size of males is a result of them growing for a longer period (Bradbury, 1977a). Given that males need to achieve a larger size, delayed maturity is an obvious consequence. Sexual differences in the abandonment of reproductive effort are less easily explained through body size differences. $A$ priori, one might expect the smaller sex (in this case females), to be more sensitive to ambient temperature fluctuations than the larger sex. The converse is the case. In examining those factors which might modulate the undertaking of reproductive activities in the two sexes, we have again turned to foraging behaviour. Since both sexes use the same diurnal roosts, predation and microclimatic effects during the day are unlikely candidates as direct causes of sexual differences in reproductive effort. As I shall argue below, distinct differences in foraging behaviour exist between the sexes and these are useful in explaining differences in reproductive timing.

Nearly $88 \%$ of the breeding season diet of Hypsignathus consists of the fruit from two genera of plants. The predominant components are fruit from the species of the genus Anthocleista. These species are all small- to medium-sized trees which grow along rivers or in secondary forest. Several species tend to form local monospecific stands of trees in different stages of growth. During the Hypsignathus mating seasons, nearly all adult Anthocleista are bearing thousands of fruits. Less than $1 \%$ of these fruits are ripe at any one time. Individual trees continue to bear fruit for many months at a time and therefore constitute known sources of food. Radio-tracked Hypsignathus appear to visit the same stands of Anthocleista night after night in a pattern which has been called "traplining". Assuming that ripe fruit are of higher nutritional value than green fruit, this foraging pattern has the benefit that the location of food is known in advance, but the detriment that most of it is of low quality.

The second major component of the Hypsignathus diet consists of fruit of the genus Ficus. In Gabon, nearly all Ficus are strangling figs with small fruit crop biomasses. Unlike Anthocleista, Ficus plants bear ripe fruit for only about 10 days at a time and do not refruit for intervals of from 6 months to 2 years (G. Michaloud, personal communication). Also unlike Anthocleista, Ficus do not form monospecific stands but are randomly distributed through the mainland forest. When feeding on Ficus, radio-tracked Hypsignathus return to the same tree night after night until its fruit is exhausted. Figs are apparently hard to find as evidenced by the fact that several radio-tracked Hypsignathus missed trees in fruit near to their day roosts and flew much further to feed than was necessary. (Trees in fruit are not defended against conspecifics by these bats). The difficulty in finding currently ripe Ficus fruit presumably arises from the short duration of ripe fruit on a given tree, the long intervals between refruitings, the asynchrony in fruiting among nearby trees, and the random spatial dispersion of the trees. Once located, however, a Ficus in fruit provides a substantial supply of ripe fruit of high quality. 
Major differences between the sexes in foraging have been demonstrated using radio-tracking and by collecting faeces in the field. Female and young male Hypsignathus forage at distances of 2-5 km from their day roosts and strongly favour Anthocleista as a food over Ficus. Adult males forage at distances of 6-12 km from their day roosts and eat a significantly larger amount of Ficus. The differences in foraging ranges appear to follow from the differences in diet in that we might expect bats to hunt over a larger area to find a Ficus than to trapline a patch of known Anthocleista.

These differences can be interpreted as follows. Females and young have adopted a conservative energy strategy. They forage preferentially on a food supply which is easily found on successive nights, but which is of low average quality. Put another way, the average food input is of low value, but it has a low variance over time. For a female which can produce only a single young each season, or young males which are focused on survival instead of reproduction, avoidance of food shortages may be more important than enjoyment of occasional food surpluses. Adult males have taken on a more opportunistic and risky energetic strategy. They have opted for a food which is hard to find on a day-to-day basis, but which can provide a local short-term energy boost when found. The chance that such a boost will be found is presumably the reason for this dietary bias. As noted, the skew in male mating success on Hypsignathus leks is acute and over $70 \%$ of the displaying males may obtain no copulations in a whole season. Any factor which might enhance a male's displaying performance and hence its reproductive success could well be worth the risks. And the risks seem to be appreciable. The high variance in foraging success when utilizing Ficus is presumably a major cause of the asynchronous abandonment of display by adult males. That adult males are playing a dangerous energy game is also indicated by the synchronous abandonment of display during periods of cold weather and by the higher mortality rates of adult males (about $45 \%$ per year) when compared to females or juvenile males (about $37 \%$ per year). Finally, it may even be the case that the larger size of adult males (and hence the longer maturation period) is not the result of agonistic or display competition on the lek, but is instead a means of increasing mobility and reducing relative energy costs through classical allometric relations. Direct measurements on the relative energy costs of food-stressed male and female Hypsignathus would be of great interest.

To summarize this section, I have tried to argue that the striking intersexual differences in timing of reproductive effort in Hypsignathus can be plausibly related to (a) the relative expectations of reproductive success of each sex given the lek mating system, (b) the opportunity to modify these expectations by choice of diets with either low or high variance in foraging success, and (c) differences in adult body sizes arising either directly from mating behaviour or indirectly through sexually different energy strategies. Since so many bats appear to have polygynous or promiscuous mating systems, have access to a range of foods, and show some sexual dimorphism in size (see Bradbury, 1977b), it is possible that similar factors and relationships have led to differences between the sexes in reproductive timing throughout the group.

\section{Intrasexual diversity in timing}

In the section above, it was argued that stochastic variations in foraging success led to variations in social status, especially for males, and thence reproductive success. In this section, I shall outline some examples in which the causal sequence is reversed and deterministic: that is, in which current social status directly determines access to foraging sites and this in turn determines reproductive success. Both examples are drawn from the same group of emballonurids discussed in the first section.

In the small emballonurid, Rhynchonycteris naso, nearly all adult-sized members of colonies are recruits from other nearby groups (Bradbury \& Vehrencamp, 1976). Curiously, all of our 
study groups included one or two female members which were adult-sized, had never before borne young (as evidenced by teat size), and which failed to produce a single youngster in over a year's observations. This was the case even though all other females in the colonies produced at least one young and some produced two successive young in the same period. While it would not be surprising to find delayed maturity in youngsters retained within their parental units (e.g. $S$. leptura), it is not at all clear why these young $R$. naso females should defer reproduction in favour of unrelated females. That they are simply not yet sexually mature seems unlikely because sympatric and larger emballonurids typically first mate at 6 months and bear young as yearlings.

One possible answer has arisen as the result of direct observations on foraging by this species (Bradbury \& Vehrencamp, 1976; J. Bradbury, unpublished observations). Each $R$. naso colony forages within a 1 hectare territory over a stream or river. The adult females which breed and at least one adult male typically forage as a cohesive flock over some central portion of this territory. The nonbreeding females and the other males forage singly, over a wider area per bat, and in the more peripheral parts of the colony territory. When recent young are present in the colony, they forage with their mothers in the central 'core' area, and not with the nonbreeding females at the periphery. Our initial samples of aerial insects at several points within the territory of a medium-sized colony in 1976 showed that insect densities were typically higher in the core areas favoured by the breeding females than in the peripheral areas used by nonbreeding females. It is extremely tempting to suggest that differential access to food supplies determines the differential undertaking of pregnancies in $R$. naso females. To date, we have not seen any defence of core areas by breeding females (or possibly males) but, given the compositional stability of these colonies, dominance relations might be expected to be stable for long periods and challenges to status infrequent.

As a second case of deterministic differences in reproductive effort within a sex, I return to the emballonurid Saccopteryx bilineata. Colonies of this species are divided up into harems and solitary males. At the day roost, most males defend a small area of vertical surface by means of a rich repertoire of visual, vocal and olfactory displays. Females roost with certain males to form harems. At night, this social structure is mapped directly onto the foraging grounds with each male defending a territory within which his current females feed (Bradbury \& Vehrencamp, 1976).

Amongst males, there appear to be several different methods of harem acquisition. Roughly $24 \%$ of all male offspring disperse to other colonies and set up roost territories. The remaining $76 \%$ either set up territories in the parental colony or remain within the territory of their fathers in a sexually inactive condition. These latter were called "crypto males" by Tannenbaum (1975) in consequence of their markedly low profiles in the parental unit. Only a small fraction of the young adopt crypto-male behaviour in a given year. Of the males which set up territories, most do not obtain females in their first year. While a considerable exchange of females between males occurs during a normal seasonal sequence, these exchanges are restricted to a small subset of the territorial males in a given colony (Bradbury \& Vehrencamp, 1976). The other 'peripheral' territorial males rarely if ever obtain females until they can succeed to the territories of a more successful male. About $40 \%$ of the territorial males in a colony control females at a given time and only $30 \%$ of the peripheral males can expect to become harem masters each year (Tannenbaum, 1975).

The factor controlling this distribution of males is most probably the number and quality of foraging sites. Considerable exchange of females occurs each year between harem males, even though the conditions at the day roost show minimal seasonal variation. This suggests that it is the quality of successive male foraging territories which determines the size and membership of harems (Bradbury \& Vehrencamp, 1977a). As current harem males die, their harems and territories are taken over by crypto males from within or nearby peripheral males from without (Tannenbaum, 1975). Age seems an important factor in determining the sequence of accession to these limited foraging territories. Thus, as appears to be the case in female $R$. naso, age and 
status in the social milieu at the day roost determines which males of $S$. bilineata control the optimum foraging sites and thus which obtain the highest reproductive success. The number and distribution of each type of foraging site are relatively stable parameters from year to year and are the probable determinants of the fixed ratios of each social strategy found in typical colonies.

\section{Conclusions}

I hope the preceding examples will have justified the following three points. First, in our search for general rules governing the synchronization of reproductive cycles with climatic cycles or with phylogenetic affinities, we must not forget that there exists a considerable amount of variation around each mean pattern. Rather than ignore this variation, any predictive theories should be broad enough to explain the exceptions and heterogeneities as well as the convergences. In many cases, the exceptions may provide useful insights into the means by which the typical patterns are generated.

Secondly, differences at finer levels constitute especially fertile ground for co-operative studies by physiologists, field ecologists and social ethologists. The causes and consequences of social status are a critical issue in any sociobiological theory and many of these are physiological effects mediated through ecological factors. It would be of great interest to know, for example, how food mediates the shift in reproductive states for female Rhynchonycteris or male Hypsignathus. What levels of food stress trigger resorption/abortion in $S$. bilineata females and how do $B$. plicata females avoid similar fetal loss rates? One aim of this paper is to encourage more joint studies at this level in bats.

Finally, it is interesting to me how most of the examples above show a similar pattern of interplay between foraging behaviour, demographic and energy strategies, and reproductive timing. This common theme may simply be a result of the small sample or my own prejudices. Whether so or not, I hope it constitutes a challenge to other field workers to examine reproductive cycles at the same levels, and to tease out alternative patterns in other groups. It is often easier to design studies when there are specific models to disprove. We have advanced a few and we hope it encourages further discussion and research.

\section{References}

Bradbury, J.W. (1977a) Lek mating behavior in the hammer-headed bat. Z. Tierpsychol. 45, 225-255.

Bradbury, J.W. (1977b) Social organization and communication. In The Biology of Bats, Vol. III, pp. 1-72. Ed. W. Wimsatt. Academic Press, New York,

Bradbury, J.W. \& Vehrencamp, S.L. (1976) Social organization and foraging in emballonurid bats. I. Field studies. Behav. Ecol. Sociobiol. 1, 337-381.

Bradbury, J.W. \& Vehrencamp, S.L. (1977a) Social organization and foraging in emballonurid bats. III. Mating systems. Behav. Ecol. Sociobiol. 2, 1-17.

Bradbury, J.W. \& Vehrencamp, S.L. (1977b) Social organization and foraging in emballonurid bats. IV. Parental investment patterns. Behav. Ecol. Sociobiol. 2, 19-29.

Bradshaw, G.V.R. (1962) Reproductive cycle of the California leaf-nosed bat, Macrotus californicus. Science, N.Y. 136, 645-646.

Constantine, D.G. (1967) Activity patterns of the Mexican free-tailed bat. Univ. New Mex. Publs Biol. 7, 8-79.
Goodman, D. (1974) Natural selection and a cost ceiling on reproductive effort. Am. Nat. 108, 247-268.

Lopez-Forment, W. (1976) Some ecological aspects of the bat Balantiopteryx plicata plicata Peters, 1867 (Chiroptera: Emballonuridae) in Mexico. M.S. thesis, Cornell University, Ithaca, New York.

Ramaswamy, K.R. (1961) Studies on the sexual cycle of the Indian vampire bat, Megaderma lyra lyra. I. Breeding habits. Proc. natn. Inst. Sci. India, B 27, 287-307.

Schaffer, W.M. (1974) Selection for optimal life histories: the effects of age structure. Ecology 55, 291303.

Short, H.L. (1961) Age at sexual maturity of Mexican free-tailed bats. J. Mammal. 42, 533-536.

Stebbings, R.E. (1966) A population study of bats of the genus Plecotus. J. Zool., Lond. 150, 53-75.

Tannenbaum, B. (1975) Reproductive strategies in the while-lined bat. Ph.D. thesis, Cornell University, Ithaca. New York. 